\title{
Non-dipole effects in the angular distribution of photoelectrons in sequential two-photon atomic double ionization
}

\author{
A N Grum-Grzhimailo ${ }^{1}$, E V Gryzlova ${ }^{1}$, and M Meyer ${ }^{2}$ \\ ${ }^{1}$ Skobeltsyn Institute of Nuclear Physics, Lomonosov Moscow State University, \\ Moscow 119234, Russia \\ ${ }^{2}$ European XFEL GmbH, Albert-Einstein-Ring 19, D-22761 Hamburg, Germany \\ E-mail: algrgr1492@yahoo.com
}

\begin{abstract}
Motivated by the recent achievements of experiments using X-ray free electron lasers, we develop a theory for the angular distributions of photoelectrons in sequential two-photon double ionization (2PDI) of atoms beyond the dipole approximation. Expressions for the angular distributions are obtained taking into account the full multipole expansion of radiation in electric and magnetic moments. The formalism is further specified for the first-order corrections to the dipole approximation. As illustrative example, the sequential 2PDI of the $2 \mathrm{p}$ shell in atomic neon is studied. The numerical calculations predict distinct non-dipole effects observable in experiments at present X-ray Free Electron Lasers.
\end{abstract}

PACS numbers: $32.80 . \mathrm{Fb}, 32.80 . \mathrm{Rm}$

Submitted to: J. Phys. B: At. Mol. Opt. Phys. 


\section{Introduction}

With the advent of free electron lasers (FELs) generating intense short pulses of XUV and X-ray radiation, studies of non-linear photoprocesses in this wavelength range became feasible [1]. These studies have fundamental importance for our understanding of the interaction between intense high-frequency fields and quantum systems. Investigations of atomic ionization by a few photons reveal basic features of non-linear processes in the short wavelength regime. When the photon energy is larger than the ionization potential of the singly charged ion, the sequential ionization is generally the dominant non-linear mechanism involving the outer atomic shells $[2,3]$. In the sequential two-photon double ionization (2PDI), which is one of the simplest non-linear atomic processes in the XUV, the system evolves after emission of the first electron until the singly charged (intermediate) ion absorbs one more photon from the same pulse.

The conventional theory of non-linear photoprocesses is developed within the dipole $E 1$ approximation, as presented in numerous textbooks and reviews (for example $[4,5,6,7])$. The dipole approximation is, with very rare exceptions, justified for the optical and near UV range. Studies of non-linear non-dipole phenomena in atomic ionization are up to now also restricted to a special case of resonant ionization in the optical regime, when the photon frequency is in resonance with a discrete quadrupole $E 2$ transition [8, 9, 10, 11]. The theory of the sequential ionization, developed so far also within the dipole approximation $[12,13,14]$, has been used rather successfully in explaining the first results obtained in the corresponding experiments with FELs $[15,16,17,18,19,20,21,22]$. The energies of the photons in the above experiments were not high enough to introduce non-dipole contributions. However, it is known that already at photon energies of a few hundreds $\mathrm{eV}$ and sometimes even lower, the photoelectron angular distributions in atomic single-photon ionization can be affected significantly by interference between the electric dipole and electric quadrupole amplitudes [23, 24, 25]. Going beyond the dipole approximation became a routine theoretical procedure in the XUV angle-resolved photoelectron spectroscopy (ARPES) in the gas phase. Similar non-dipole transitions to continuum are involved in linear and non-linear photoprocesses. Thus, the non-dipole effects will be present also in the XUV/X-ray non-linear atomic processes, accessible now with FELs. It is the main goal of the present paper to extend the theory of the sequential 2PDI by incorporating higher multipoles of the radiation field. In Section 2 we give the theoretical grounds for treating the non-dipole effects in the sequential 2PDI. In Section 3 the formalism is specified by taking into account first-order non-dipole corrections in the photoelectron angular distributions. Section 4 presents numerical examples for the sequential 2PDI of the neon atom in the photon energy range between 100 and $1100 \mathrm{eV}$.

Atomic units are used throughout until otherwise indicated. 


\section{Basic formalism}

We consider the sequential 2PDI of an atom as a two-step process. At the first step, the atom is singly ionized by the first photon $\hbar \omega_{1}$ which produces an ionic state through emission of the first electron $e_{1}$,

$$
\hbar \omega_{1}+\mathrm{A}\left(\alpha_{0} J_{0}\right) \rightarrow \mathrm{A}^{+}\left(\alpha_{i} J_{i}\right)+e_{1}\left(l_{1} j_{1}\right) .
$$

The first step leads, in general, to the polarized intermediate ionic state $A^{+}\left(\alpha_{i} J_{i}\right)$. At the second step, the ion is further ionized by the second photon, $\hbar \omega_{2}$, with emission of the second electron $e_{2}$,

$$
\hbar \omega_{2}+\mathrm{A}^{+}\left(\alpha_{i} J_{i}\right) \rightarrow \mathrm{A}^{++}\left(\alpha_{f} J_{f}\right)+e_{2}\left(l_{2} j_{2}\right) .
$$

Here $J_{0}, J_{i}$ and $J_{f}$ are total angular momenta of the neutral atom, the intermediate singly charged ion and the final doubly charged ion, respectively; $\alpha_{0}, \alpha_{i}, \alpha_{f}$ are the sets of other quantum numbers needed to specify the corresponding state. We will omit the latter in further expressions for brevity. The emitted electrons are described by their orbital $(l)$ and total $(j)$ angular momenta.

The derivation for the photoelectron angular distributions proceeds within the standard statistical tensor formalism [26] and in close similarity to [13]. In this section we will, where possible, maintain the notations of [13], generalizing it for the full multipole expansion of radiation in electric and magnetic moments. The statistical tensors describing the intermediate single-charged ionic state depend on the photoelectron $\left(e_{1}\right)$ emission angles $\vartheta_{1}$ and $\varphi_{1}$. They can be cast into the form (compare to equation (5) of $[13])$

$$
\begin{aligned}
& \rho_{k_{i} q_{i}}\left(J_{i}, J_{i}^{\prime} ; \vartheta_{1}, \varphi_{1}\right)=\pi \alpha \omega_{1}\left(2 J_{0}+1\right)^{-\frac{1}{2}} \\
& \quad \times \sum_{\substack{k_{1} q_{1} k_{\gamma_{1}} q_{\gamma_{1}} \\
\pi_{1} L_{1} \pi_{1}^{\prime} L_{1}^{\prime}}}\left(k_{i} q_{i}, k_{1} q_{1} \mid k_{\gamma_{1}} q_{\gamma_{1}}\right) B^{\pi_{1} L_{1}, \pi_{1}^{\prime} L_{1}^{\prime}}\left(k_{1}, k_{i}, k_{\gamma_{1}}\right) \\
& \quad \times \rho_{k_{\gamma_{1}} q_{\gamma_{1}}}\left(\pi_{1} L_{1}, \pi_{1}^{\prime} L_{1}^{\prime}\right) \sqrt{\frac{4 \pi}{2 k_{1}+1}} Y_{k_{1} q_{1}}\left(\vartheta_{1}, \varphi_{1}\right),
\end{aligned}
$$

where $\alpha$ is the fine-structure constant and $Y_{k q}(\theta, \phi)$ denotes the spherical harmonic in the Condon-Shortley phase convention; we use the standard notation for the ClebschGordon coefficients. The indices $\pi_{1}, \pi_{1}^{\prime}$ take values of 0 or 1 for the electric and magnetic multipoles, respectively; $L_{1}$ and $L_{1}^{\prime}$ denote the photon $\hbar \omega_{1}$ multipolarity. General expressions for the photon statistical tensors $\rho_{k_{\gamma} q_{\gamma}}\left(\pi L, \pi^{\prime} L^{\prime}\right)$ in terms of the Stokes parameters of the radiation were presented, for example, in [27]. The dynamical coefficients $B^{\pi_{1} L_{1}, \pi_{1}^{\prime} L_{1}^{\prime}}\left(k_{1}, k_{i}, k_{\gamma_{1}}\right)$ in equation (3) depend on the partial multipole amplitudes $T_{J_{0}, J_{i} l_{1} j_{1} J_{1}}^{\pi_{1} L_{1}} \equiv\left\langle\alpha_{i} J_{i} l_{1} j_{1} J_{1}\left\|\hat{T}^{\pi_{1} L_{1}}\right\| \alpha_{0} J_{0}\right\rangle$, where $\boldsymbol{J}_{1}=\boldsymbol{J}_{i}+\boldsymbol{j}_{1}$ is the total angular momentum of the system $A^{+}\left(\alpha_{i} J_{i}\right)+e_{1}\left(l_{1} j_{1}\right)$ :

$$
B^{\pi_{1} L_{1}, \pi_{1}^{\prime} L_{1}^{\prime}}\left(k_{1}, k_{i}, k_{\gamma_{1}}\right)=\frac{3 \hat{k}_{1} \hat{k}_{i}}{\hat{L}_{1} \hat{L}_{1}^{\prime}}
$$




$$
\begin{aligned}
& \times \sum_{\substack{l_{1} l_{1}^{\prime} j_{1} j_{1}^{\prime} \\
J_{1} J_{1}^{\prime}}}(-1)^{\xi_{1}} \hat{l}_{1} \hat{l}_{1}^{\prime} \hat{j}_{1} \hat{j}_{1}^{\prime} \hat{J}_{1} \hat{J}_{1}^{\prime}\left(l_{1} 0, l_{1}^{\prime} 0 \mid k_{1} 0\right) \\
& \times\left\{\begin{array}{ccc}
k_{\gamma_{1}} & J_{1} & J_{1}^{\prime} \\
J_{0} & L_{1}^{\prime} & L_{1}
\end{array}\right\}\left\{\begin{array}{ccc}
j_{1} & l_{1} & 1 \\
l_{1}^{\prime} & j_{1}^{\prime} & k_{1}
\end{array}\right\}\left\{\begin{array}{ccc}
J_{i} & j_{1} & J_{1} \\
J_{i}^{\prime} & j_{1}^{\prime} & J_{1}^{\prime} \\
k_{i} & k_{1} & k_{\gamma_{1}}
\end{array}\right\} \\
& \times T_{J_{0}, J_{i} l_{1} j_{1} J_{1}}^{\pi_{1} L_{J_{0}, J_{i}^{\prime} l_{1}^{\prime} j_{1}^{\prime} J_{1}^{\prime}},}
\end{aligned}
$$

where $\xi_{1}=J_{1}+J_{0}+k_{\gamma_{1}}+j_{1}^{\prime}+L_{1}^{\prime}+\frac{1}{2}$, the standard notations for the $6 \mathrm{j}$ and $9 \mathrm{j}$ Wigner coefficients are used and we abbreviate $\hat{a} \equiv \sqrt{2 a+1}$. It is implied that the dynamical coefficients (4) are normalized so that the zero rank tensor (3) is related to the differential cross section of the first-step photoionization, $\rho_{00}\left(J_{i}, J_{i} ; \vartheta_{1}, \varphi_{1}\right)=\hat{J}_{i}^{-1} d \sigma_{1}\left(J_{i}\right) / d \Omega_{1}$.

The angular correlation function between electrons $e_{1}$ and $e_{2}$ is the angular distribution of the second-step photoelectron $e_{2}$ emitted from the intermediate ion which polarization is described by the statistical tensors (3). Applying a theory of photoelectron angular distributions from polarized atoms for arbitrary multipoles of the radiation field [28], we obtain

$$
\begin{aligned}
& W\left(J_{f} ; \vartheta_{1}, \varphi_{1}, \vartheta_{2}, \varphi_{2}\right)=\pi \alpha \omega_{2} \\
& \quad \times \sum_{\substack{k_{2} k_{\gamma_{2}} k_{i} \\
q_{2} q_{2} q_{i}}} \sum_{\substack{\pi_{2} L_{2} \pi_{2}^{\prime} L_{2}^{\prime} \\
J_{i} J_{i}^{\prime}}} \bar{B}^{\pi_{2} L_{2}, \pi_{2}^{\prime} L_{2}^{\prime}}\left(k_{i}, k_{2}, k_{\gamma_{2}}\right) \rho_{k_{\gamma_{2}} q_{\gamma_{2}}}\left(\pi_{2} L_{2}, \pi_{2}^{\prime} L_{2}^{\prime}\right) \\
& \quad \times h\left(J_{i}, J_{i}^{\prime}\right) \rho_{k_{i} q_{i}}\left(J_{i}, J_{i}^{\prime} ; \vartheta_{1}, \varphi_{1}\right)\left(k_{i} q_{i}, k_{\gamma_{2}} q_{\gamma_{2}} \mid k_{2} q_{2}\right) \\
& \quad \times \sqrt{\frac{4 \pi}{2 k_{2}+1}} Y_{k_{2} q_{2}}\left(\vartheta_{2}, \varphi_{2}\right) .
\end{aligned}
$$

Here $\rho_{k_{\gamma_{2}} q_{\gamma_{2}}}\left(\pi_{2} L_{2} ; \pi_{2}^{\prime} L_{2}^{\prime}\right)$ are statistical tensors of the second photon, while the statistical tensors of the intermediate ion, $\rho_{k_{i} q_{i}}\left(J_{i}, J_{i}^{\prime} ; \vartheta_{1}, \varphi_{1}\right)$, are given by equation (3). The complex matrix $h\left(J_{i}, J_{i}^{\prime}\right)$ describes the depolarization of the intermediate ion in the time interval between the absorption of the first and the second photon due to the time evolution of the density matrix of the angular momentum of the electronic shell . In the present case the key reason for this evolution is the precession of the angular momentum due to the spin-orbit interaction in the electronic shell. In general, the matrix $h\left(J_{i}, J_{i}^{\prime}\right)$ can reflect arbitrary coherent properties of the excited fine-structure levels. In this sense the theory is applicable to both of the main operating modes of X-ray FELs, i.e. to the self-amplified stimulated emission (SASE) and to the seeding mode. However, it is extremely difficult to calculate the depolarization factors for real situations taking into account the microbunching, variations in the FEL pulse shape from shot to shot and the poorly known longitudinal coherence of the light from FEL. It may be more practical in certain situations to consider the depolarization factors as unknown fitting parameters as in studies of photoionization by optical lasers (for example [29, 30]). The particular cases $h\left(J_{i}, J_{i}^{\prime}\right)=\delta_{J_{i}, J_{i}^{\prime}}$ and $h\left(J_{i}, J_{i}^{\prime}\right)=1$ describe incoherent excitation of separated finestructure levels and excitation of completely overlapping (degenerate) fine-structure levels (LS-coupling limit), respectively. More details on the matrix $h\left(J_{i}, J_{i}^{\prime}\right)$ in the 2PDI 
process, including intermediate cases of coherence, can be found in [14, 31]. We return to this point for a particular example in Section 4.

The dynamical coefficients $\bar{B}^{\pi_{2} L_{2} ; \pi_{2}^{\prime} L_{2}^{\prime}}\left(k_{i}, k_{2}, k_{\gamma_{2}}\right)$ in equation (5) are given in terms of the partial multipole amplitudes of the second step ionization $T_{J_{i}, J_{f} l_{2} j_{2} J_{2}}^{\pi_{2} L_{2}} \equiv$ $\left\langle\alpha_{f} J_{f}, l_{2} j_{2}: J_{2}\left\|\hat{T}^{\pi_{2} L_{2}}\right\| \alpha_{i} J_{i}\right\rangle$ :

$$
\begin{aligned}
& \bar{B}^{\pi_{2} L_{2}, \pi_{2}^{\prime} L_{2}^{\prime}}\left(k_{i}, k_{2}, k_{\gamma_{2}}\right)=\frac{3 \hat{k}_{i} \hat{k}_{\gamma_{2}}}{\hat{L}_{2} \hat{L}_{2}^{\prime}} \\
& \times \sum_{\substack{l_{2} l_{2}^{\prime} j_{2} j_{2}^{\prime} \\
J_{2} J_{2}^{\prime}}}(-1)^{\xi_{2}} \hat{l}_{2} \hat{l}_{2}^{\prime} \hat{j}_{2} \hat{j}_{2}^{\prime} \hat{J}_{2} \hat{J}_{2}^{\prime}\left(l_{2} 0, l_{2}^{\prime} 0 \mid k_{2} 0\right) \\
& \quad \times\left\{\begin{array}{lll}
j_{2} & J_{2} & J_{f} \\
J_{2}^{\prime} & j_{2}^{\prime} & k_{2}
\end{array}\right\}\left\{\begin{array}{lll}
j_{2} & l_{2} & 1 / 2 \\
l_{2}^{\prime} & j_{2}^{\prime} & k_{2}
\end{array}\right\}\left\{\begin{array}{lll}
J_{i} & L_{2} & J_{2} \\
J_{i}^{\prime} & L_{2}^{\prime} & J_{2}^{\prime} \\
k_{i} & k_{\gamma_{2}} & k_{2}
\end{array}\right\} \\
& \times T_{J_{i}, J_{f} l_{2} j_{2} J_{2}}^{\pi_{2} L_{2}} T_{J_{i}^{\prime}, J_{f} l_{2}^{\prime} j_{2}^{\prime} J_{2}^{\prime}}^{\pi_{\prime}^{\prime} L^{\prime} *}
\end{aligned}
$$

where $\xi_{2}=J_{2}+J_{f}+k_{2}-\frac{1}{2}$ and $\boldsymbol{J}_{2}=\boldsymbol{J}_{f}+\boldsymbol{j}_{2}$ is the total angular momentum of the system $A^{++}\left(\alpha_{f} J_{f}\right)+e_{2}\left(l_{2} j_{2}\right)$. Note the following useful relations

$$
\begin{aligned}
& B^{\pi L, \pi^{\prime} L^{\prime}}\left(k, k_{i}, k_{\gamma}\right)=(-1)^{k_{i}+k+k_{\gamma}+L-L^{\prime}}\left(B^{\pi^{\prime} L^{\prime}, \pi L}\left(k, k_{i}, k_{\gamma}\right)\right)^{*}, \\
& \bar{B}^{\pi L, \pi^{\prime} L^{\prime}}\left(k_{i}, k, k_{\gamma}\right)=(-1)^{k_{i}+k+k_{\gamma}+L-L^{\prime}}\left(\bar{B}^{\pi^{\prime} L^{\prime}, \pi L}\left(k_{i}, k, k_{\gamma}\right)\right)^{*},
\end{aligned}
$$

which result from (4) and (6), respectively (the latter for a sharp value of $J_{i}$ ), upon permutation of primed and not primed quantum numbers.

To obtain the dipole approximation, only terms with $\pi_{1}=\pi_{1}^{\prime}=\pi_{2}=\pi_{2}^{\prime}=0$ and $L_{1}=L_{1}^{\prime}=L_{2}=L_{2}^{\prime}=1$ should be left in expressions (3)-(6). In this case equations (3)-(6) reduce to equations (5), (6), (12) and (13) of [13], respectively, after assuming that both photons are linearly polarized in one direction ( $z$ axis) and choosing the coordinate system with the $x$ axis in the reaction plane spanned by the $z$ axis and the linear momentum of the first electron $\left(\varphi_{1}=0\right)$.

We concentrate below on the non-coincidence type of experiments, i.e. when only one of the two photoelectrons is detected. Measurements in the coincidence mode in sequential 2PDI [16] suffer so far from low statistics caused by the low repetition rate of present X-ray FEL sources. Integrating the angular correlation function (5) over the emission angles of the first $\left(e_{1}\right)$ or the second $\left(e_{2}\right)$ electron, we obtain the photoelectron angular distribution for the electrons $e_{2}$ and $e_{1}$, respectively. After integration, only terms with $k_{1}=0$ or $k_{2}=0$ survive, respectively. It follows from the property of the Clebsch-Gordon coefficients in (4) or (6) that the angular distribution of the second (first) photoelectron is determined by terms with $l_{1}=l_{1}^{\prime}\left(l_{2}=l_{2}^{\prime}\right)$ and hence, only contributions from the field multipoles of the same parity remain for the first (second) ionization step.

The angular distribution of the electron $e_{1}$, as follows from (5), takes the form

$$
\frac{d \sigma_{1}}{d \Omega_{1}}=4 \pi^{2} \alpha \omega_{2}
$$


Non-dipole effects in sequential two-photon double ionization

$$
\begin{aligned}
& \times \sum_{\substack{k_{i} q_{i} \\
J_{i} J_{i}^{\prime}}}\left[\sum_{\substack{\pi_{2} L_{2} \\
\pi_{2}^{\prime} L_{2}^{\prime}}} \bar{B}^{\pi_{2} L_{2}, \pi_{2}^{\prime} L_{2}^{\prime}}\left(k_{i}, 0, k_{i}\right) \rho_{k_{i} q_{i}}^{*}\left(\pi_{2} L_{2}, \pi_{2}^{\prime} L_{2}^{\prime}\right)\right] \\
& \times(-1)^{k_{i}} \hat{k}_{i}^{-1} h\left(J_{i}, J_{i}^{\prime}\right) \rho_{k_{i} q_{i}}\left(J_{i}, J_{i}^{\prime} ; \vartheta_{1}, \varphi_{1}\right) .
\end{aligned}
$$

As was outlined first in [13] and further discussed in [16, 32, 33] within the dipole approximation, the angular distribution of the electrons produced in the first ionization step of the 2PDI generally differs from the photoelectron angular distribution for the same ionization transition in the single-photon ionization. It follows from the fact that despite the integration over the emission angles, the second electron is still supposed to be ejected by the second photon. Equation (9) contains information on the second ionization step in the factor in square brackets. In the particular case of an unpolarized intermediate ionic state $\left(k_{i}=0\right)$, the angular distribution of the first-step electron $e_{1}$ is not affected by the second ionization step.

The angular distribution of the electron $e_{2}$ is expressed by equation (5) with substitution of the integral statistical tensor of the intermediate ionic state,

$$
\begin{aligned}
& \rho_{k_{i} q_{i}}\left(J_{i}, J_{i}^{\prime}\right)=4 \pi^{2} \alpha \hat{J}_{0}^{-1} \\
& \quad \times \sum_{\pi_{1} L_{1} \pi_{1}^{\prime} L_{1}^{\prime}} B^{\pi_{1} L_{1}, \pi_{1}^{\prime} L_{1}^{\prime}}\left(0, k_{i}, k_{i}\right) \rho_{k_{i} q_{i}}\left(\pi_{1} L_{1}, \pi_{1}^{\prime} L_{1}^{\prime}\right),
\end{aligned}
$$

instead of the differential statistical tensor (3). Equation (10) is obtained by integrating equation (3) over the emission angles of electron $e_{1}$. Thus, the angular distribution of electron $e_{2}$ takes the form

$$
\begin{aligned}
& \frac{d \sigma_{2}}{d \Omega_{2}}=\pi \alpha \omega_{2} \\
& \quad \times \sum_{\substack{k_{2} q_{2} \\
k_{\gamma_{2}} q_{2}}} \sum_{\substack{k_{i} q_{i} \\
J_{i} J_{i}^{\prime}}}\left[\sum_{\substack{\pi_{2} L_{2} \\
\pi_{2}^{\prime} L_{2}^{\prime}}} \bar{B}^{\pi_{2} L_{2}, \pi_{2}^{\prime} L_{2}^{\prime}}\left(k_{i}, k_{2}, k_{\gamma_{2}}\right) \rho_{k_{\gamma_{2}} q_{\gamma_{2}}}\left(\pi_{2} L_{2}, \pi_{2}^{\prime} L_{2}^{\prime}\right)\right] \\
& \quad \times h\left(J_{i}, J_{i}^{\prime}\right) \rho_{k_{i} q_{i}}\left(J_{i}, J_{i}^{\prime}\right)\left(k_{i} q_{i}, k_{\gamma_{2}} q_{\gamma_{2}} \mid k_{2} q_{2}\right) \sqrt{\frac{4 \pi}{2 k_{2}+1}} Y_{k_{2} q_{2}}\left(\vartheta_{2}, \varphi_{2}\right) .
\end{aligned}
$$

Now we are in a position to specify the general formalism for particular applications.

\section{First-order non-dipole corrections}

The first-order non-dipole corrections in equations (4) and (6) are given by the $E 1-E 2$ and E1-M1 interference terms. The corresponding spherical components $(\lambda)$ of the multipole operators are (in the nonrelativistic long-wave approximation) of the form

$$
\begin{aligned}
& \hat{T}_{\lambda}^{E 1}=D_{\lambda}=\sqrt{\frac{4 \pi}{3}} \sum_{n} r_{n} Y_{1 \lambda}\left(\theta_{n}, \phi_{n}\right), \\
& \hat{T}_{\lambda}^{E 2}=\frac{i \alpha \omega}{2 \sqrt{3}} Q_{\lambda}=\frac{i \alpha \omega}{2 \sqrt{3}} \sqrt{\frac{4 \pi}{5}} \sum_{n} r_{n}^{2} Y_{2 \lambda}\left(\theta_{n}, \phi_{n}\right),
\end{aligned}
$$


Non-dipole effects in sequential two-photon double ionization

$$
\hat{T}_{\lambda}^{M 1}=-i M_{\lambda}=-\frac{i \alpha}{2} \sum_{n}\left(\hat{l}_{\lambda, n}+2 \hat{s}_{\lambda, n}\right),
$$

where $D_{\lambda}, Q_{\lambda}$ and $M_{\lambda}$ are the components of the atomic electric-dipole, electricquadrupole and magnetic-dipole momenta, respectively, $r_{n}, \theta_{n}$ and $\phi_{n}$ are spherical coordinates of the atomic electrons, $l_{\lambda, n}$ and $s_{\lambda, n}$ are the components of the orbital and spin angular momentum of the $n$th atomic electron, and summation is performed over all atomic electrons. In accordance with the discussion in the previous section, the E1$E 2$ and $E 1-M 1$ interference terms contribute only to the first (second) ionization step when the corresponding photoelectron $e_{1}\left(e_{2}\right)$ is observed, while the other electron is not detected.

The integral statistical tensor of the intermediate ion (10) takes the form

$$
\begin{gathered}
\rho_{k_{i} q_{i}}\left(J_{i}, J_{i}^{\prime}\right)=\frac{4 \pi^{2} \alpha \omega_{1}}{\hat{J}_{0}} \delta_{k_{i} k_{\gamma_{1}}} \delta_{q_{i} q_{\gamma_{1}}} \rho_{k_{\gamma_{1} q_{\gamma_{1}}}}(E 1, E 1) \hat{k}_{\gamma_{1}} \\
\times \sum_{\substack{l_{1} j_{1} \\
J_{1} J_{1}^{\prime}}}(-1)^{\chi} \hat{J}_{1} \hat{J}_{1}^{\prime}\left\{\begin{array}{ccc}
k_{\gamma_{1}} & J_{1} & J_{1}^{\prime} \\
J_{0} & 1 & 1
\end{array}\right\}\left\{\begin{array}{ccc}
j_{1} & J_{1} & J_{i} \\
k_{\gamma_{1}} & J_{i}^{\prime} & J_{1}^{\prime}
\end{array}\right\} \\
\times D_{J_{0}, J_{i} l_{1} j_{1} J_{1}} D_{J_{0}, J_{i}^{\prime} l_{1} j_{1} J_{1}^{\prime}}^{*}
\end{gathered}
$$

$\left(\chi=J_{1}+J_{1}^{\prime}+J_{0}+j_{1}+J_{i}+1\right)$, which is not influenced by the first-order non-dipole corrections. Here $D_{J_{0}, J_{i} l_{1} j_{1} J_{1}}=\left\langle\alpha_{i} J_{i} l_{1} j_{1} J_{1}\|D\| \alpha_{0} J_{0}\right\rangle$ is the partial dipole amplitude. Equation (15) coincides up to a not relevant normalization factor with the known expression in the dipole approximation (see equations (2.163), (2.164) of [26]). For closed-shell atoms $\left(J_{0}=0\right)$, equation (15) can be further simplified because of fixed values for $J_{1}=J_{1}^{\prime}=1$.

Substituting (15) into (11) and limiting the summation over the field multipoles to the $E 1-E 1, E 1-E 2, E 2-E 1, E 1-M 1$, and $M 1-E 1$ terms, the expression for the angular distribution of the second electron in the sequential 2PDI within the first-order nondipole corrections can be obtained. To specify this expression for the experimental conditions of measurements with FELs, we imply that both XUV photons, $\hbar \omega_{1}$ and $\hbar \omega_{2}$, belong to the same pulse of linearly polarized radiation. We choose the $x$-axis along the photon beam propagation direction and the $z$-axis along its polarization vector. The following statistical tensors of photons remain nonvanishing in this coordinate system [27]:

$$
\begin{aligned}
& \rho_{00}(E 1, E 1)=\frac{1}{\sqrt{3}} ; \rho_{20}(E 1, E 1)=-\sqrt{\frac{2}{3}} ; \\
& \rho_{1 \pm 1}(E 1, M 1)=\mp \frac{1}{2} ; \rho_{2 \pm 1}(E 1, M 1)=\frac{1}{2} ; \\
& \rho_{1 \pm 1}(E 1, E 2)= \pm \frac{1}{2} ; \rho_{2 \pm 1}(E 1, E 2)=\frac{\sqrt{5}}{6} ; \rho_{3 \pm 1}(E 1, E 2)=\mp \frac{2}{3} .
\end{aligned}
$$

The tensors with interchanged arguments are found from the general relation $\rho_{k q}\left(\pi L, \pi^{\prime} L^{\prime}\right)=(-1)^{L^{\prime}-L+q} \rho_{k-q}^{*}\left(\pi^{\prime} L^{\prime}, \pi, L\right)$. After straightforward transformations the 
angular distribution (11) of the second-step electrons $e_{2}$ within the first-order non-dipole corrections can be cast into the form

$$
\begin{aligned}
\frac{d \sigma_{2}}{d \Omega_{2}} & =\frac{\sigma_{2}}{4 \pi}\left(1+\beta_{2}^{(2)} P_{2}\left(\cos \vartheta_{2}\right)+\beta_{4}^{(2)} P_{4}\left(\cos \vartheta_{2}\right)\right. \\
& \left.\left.+\left(\delta^{(2)}+\gamma_{2}^{(2)} \cos ^{2} \vartheta_{2}+\gamma_{4}^{(2)} \cos ^{4} \vartheta_{2}\right) \sin \vartheta_{2} \cos \varphi_{2}\right)\right),
\end{aligned}
$$

where the superscript '(2)' indicates the second electron. The first three terms in equation (19) correspond to the pure dipole contribution; the last three terms give the first-order non-dipole corrections and include $E 1-E 2$ and $E 1-M 1$ interference. The last term with $\gamma_{4}$ is a non-dipole correction specific for the two-photon ionization. Neglecting polarization of the intermediate ion leads to vanishing $\beta_{4}$ and $\gamma_{4}$, and reduces equation (19) to the well-known form [34]

$$
\frac{d \sigma_{2}}{d \Omega_{2}}=\frac{\sigma_{2}}{4 \pi}\left(1+\beta_{2}^{(2)} P_{2}\left(\cos \vartheta_{2}\right)+\left(\delta^{(2)}+\gamma_{2}^{(2)} \cos ^{2} \vartheta_{2}\right) \sin \vartheta_{2} \cos \varphi_{2}\right)
$$

for the photoelectron angular distributions in single-photon ionization within the firstorder non-dipole corrections.

An equation similar to (19) describes the angular distribution of the first electron $e_{1}$ :

$$
\begin{aligned}
\frac{d \sigma_{1}}{d \Omega_{1}} & =\frac{\sigma_{1}}{4 \pi}\left(1+\beta_{2}^{(1)} P_{2}\left(\cos \vartheta_{1}\right)+\beta_{4}^{(1)} P_{4}\left(\cos \vartheta_{1}\right)\right. \\
& \left.\left.+\left(\delta^{(1)}+\gamma_{2}^{(1)} \cos ^{2} \vartheta_{1}+\gamma_{4}^{(1)} \cos ^{4} \vartheta_{1}\right) \sin \vartheta_{1} \cos \varphi_{1}\right)\right) .
\end{aligned}
$$

Note that the first-order nondipole corrections do not contribute to the factor in the square brackets in equation (9). Expressions for the parameters in equations (19) and (21) in terms of the dynamical coefficients (4), (6) are given in Appendix.

\section{Sequential 2PDI of neon}

As an example, we consider 2PDI of the neon atom:

$$
\begin{aligned}
& \hbar \omega_{1}+\mathrm{Ne}\left(2 p^{6}{ }^{1} S\right) \rightarrow \mathrm{Ne}^{+}\left(2 p^{5}{ }^{2} P\right)+e_{1} ; \\
& \hbar \omega_{2}+\mathrm{Ne}^{+}\left(2 p^{5}{ }^{2} P\right) \rightarrow \mathrm{Ne}^{++}\left(2 p^{4}{ }^{3} P,{ }^{1} D,{ }^{1} S\right)+e_{2} .
\end{aligned}
$$

The first step of the process, the atomic single-photon ionization (22), has been studied extensively over a long time (see references in [35]). These experiments were performed with synchrotron radiation from storage rings, thus due to the low intensity of the photon flux the probability of the second-step ionization (23) was negligible. Nevertheless, pronounced non-dipole effects in single ionization revealed in these experiments [36, 37] point to potentially strong non-dipole effects in the 2PDI at the corresponding photon energies. Using the Free-electron LASer in Hamburg (FLASH), the angular distributions of the individual electrons $e_{1}$ and $e_{2}$ in the 2PDI process (22), (23) were measured in $[15,22]$ while the angular correlation function between the two electrons was observed in [16]. The sequential mechanism of the 2PDI in neon was also confirmed in recoil-ion momentum distribution measurements [38] and by means of angle-resolved 


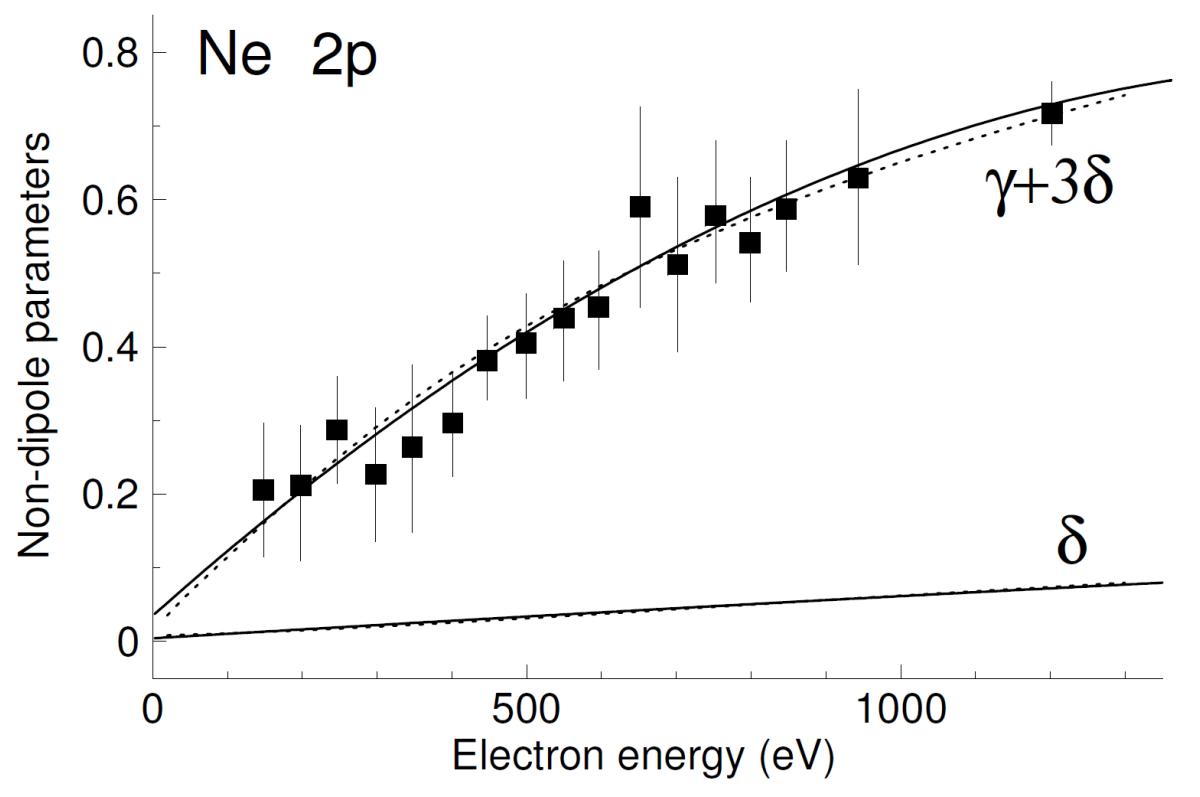

Figure 1. Non-dipole parameters $\gamma+3 \delta$ and $\delta$ of the photoelectron angular distribution in single-photon $2 \mathrm{p}$ ionization of $\mathrm{Ne}$ as a function of the photoelectron energy. Solid curves: present calculation; dotted curves: random phase approximation (RPA) [40, 41]. Experimental data of [37] for the geometry suppressing the secondorder non-dipole effects.

photoelectron spectroscopy [20]. The energies of the photons from FLASH were tens of $\mathrm{eV}$ in the above experiments and the corresponding theoretical analysis was done in the framework of the dipole approximation $[12,13,16,39]$. In the following we extend the calculations to higher photon energies and account for the first-order non-dipole corrections.

The electron wave functions to describe the first ionization step (22) were the same as in the recent multiconfiguration Hartree-Fock (MCHF) calculations [13]. Besides the 2 p emission into $s$ and $d$ continua, we added atomic continua with different parity (i.e. $p$ and $f$ waves of the photoelectron) to obtain the first-order non-dipole corrections. Since the dominant non-dipole contribution comes from the interference of the $E 1$ and $E 2$ photoionization amplitudes, we neglect the $M 1$ photoionization amplitudes (which strictly vanish in the nonrelativistic single-configuration approximation). Our results for the photoelectron angular distribution in single-photon ionization are in a good agreement with other calculations [34, 40, 41] and experiment [37] (Figure 1; we denote the non-dipole parameters in one-photon single ionization without superscript). Note that, as revealed by Derevianko et al [41], the second-order non-dipole effects start to noticeably influence the photoelectron angular distribution of the Ne $2 p$ ionization at the photon energies near to $1 \mathrm{keV}$.

For the description of the second ionization step (23), we first optimized the $1 s, 2 s$, and $2 p$ orbitals for each term of the residual ion $\mathrm{Ne}^{++}\left(2 p^{4}\right)$. These orbitals were then frozen in single-configuration $2 p^{4}\left(L_{f} S_{f}\right) E l_{2}: L_{t_{2}} S_{t_{2}}$ calculations of the $\mathrm{Ne}^{+}$ 
continuum wave functions, where $E$ and $l$ denote energy and orbital momentum of the photoelectron, $L_{t_{2}}$ and $S_{t_{2}}$ are the total orbital and spin angular momenta of the system $\mathrm{Ne}^{++}\left(2 p^{4} L_{f} S_{f}\right)+E l_{2}$, respectively, $L_{f} S_{f}$ specify the term of the $\mathrm{Ne}^{++}\left(2 p^{4}\right)$ configuration. The ionic $\mathrm{Ne}^{+}\left(2 p^{5}\right)$ state was described by mixing of configurations with the same frozen $1 s, 2 s, 2 p$ orbitals and the pseudo-orbitals $3 \bar{s}, 3 \bar{p}, 3 \bar{d}$ optimized on the ground ionic state in the multiconfiguration $1 s^{2} 2 s^{2}\left(2 p^{5}+2 p^{4} 3 \bar{p}+2 p^{3} 3 \bar{s}^{2}+2 p^{3} 3 \bar{p}^{2}+\right.$ $\left.2 p^{3} 3 \bar{d}^{2}+2 p^{3} 3 \bar{s} 3 \bar{d}\right)^{2} P$ calculation.

Calculations of the photoelectron angular distributions were performed for both intermediate fine-structure ionic states $\mathrm{Ne}^{+}\left(2 p^{5}{ }^{2} P_{1 / 2},{ }^{2} P_{3 / 2}\right)$ and for the three terms of the residual doubly charged ion $\mathrm{Ne}^{++}\left(2 p^{4}{ }^{1} S,{ }^{1} D,{ }^{3} P\right)$. The fine structure levels of the ion $\mathrm{Ne}^{++}\left(2 p^{4}{ }^{3} P_{0,1,2}\right)$ and of the intermediate ionic doublet $\mathrm{Ne}^{+}\left(2 p^{5}{ }^{2} P_{1 / 2,3 / 2}\right)$ with energy splitting of $0.114 \mathrm{eV}$ and $0.097 \mathrm{eV}$, respectively, are not resolved in current experiments using FEL radiation. Therefore, all numerical results are summed over these unresolved fine structure states. Here we assume that the fine structure states of the intermediate ion are excited incoherently, i.e. $h\left(J_{i}, J_{i}^{\prime}\right)=\delta_{J_{i} J_{i}^{\prime}}$ in equations (9), (11). This choice is made in accordance with the analysis of the $e_{1}$ and $e_{2}$ angular correlations [16, 39] in the 2PDI process (22), (23) measured with FLASH operating in the SASE mode. This analysis indicated better agreement of the measurements, although with low statistics, with the assumption of the incoherent excitation of the $\mathrm{Ne}^{+}\left(2 p^{5}{ }^{2} P_{1 / 2,3 / 2}\right)$ fine structure states. Accounting for (partial) coherence can modify the angular distribution due to other values of $h\left(J_{i}, J_{i}^{\prime}\right)$. However, we do not expect for the 2PDI (22), (23), where the intermediate ion polarization is small and smoothly depends on the photon energy (see below), significant changes in the behavior of the nondipole parameters in the noncoincidence experiments.

The three multiplet $\mathrm{Ne}^{++}\left({ }^{3} P,{ }^{1} D,{ }^{1} S\right.$, $)$ states are splitted by $3.1 \mathrm{eV}$ and $3.7 \mathrm{eV}$, respectively, which can be resolved at photon energies up to $\sim 600-700 \mathrm{eV}$ with a photon energy bandwidth of about $0.5 \%$ for the FEL pulses in SASE mode [42]. With the installation of seeding schemes in the nearest future [43] or by using monochromators [44], the energy resolution can be significantly improved.

Our numerical calculations predict that the influence of the second step ionization on the nondipole parameters of the angular distribution of the first-step photoelectrons $e_{1}$ smears out to a large extent after the angular distributions are summed over the final ionic multiplet states of $\mathrm{Ne}^{++}\left(2 p^{4}\right)$ (i.e. over energies of the unobserved electron $\left.e_{2}\right)$. This means that in case of Ne the non-dipole contributions to the first ionization step are only accessible by very demanding coincidence experiments. For this reason we concentrate in the present paper on the angular distribution of the photoelectrons $e_{2}$.

Figure 2 shows the parameters characterizing the angular distribution of the second electron $e_{2}$ in the sequential 2PDI (22), (23). The energy dependence of the dipole parameters $\beta_{2}^{(2)}$ and $\beta_{4}^{(2)}$ obtained in $[12,13]$ is extended here to higher energies (two lower panels of figure 2). Although the calculations were performed using the general equations (A.6)-(A.10) from the Appendix, it is instructive for the discussion to simplify these expressions within the single-configuration approximation and by using term- 


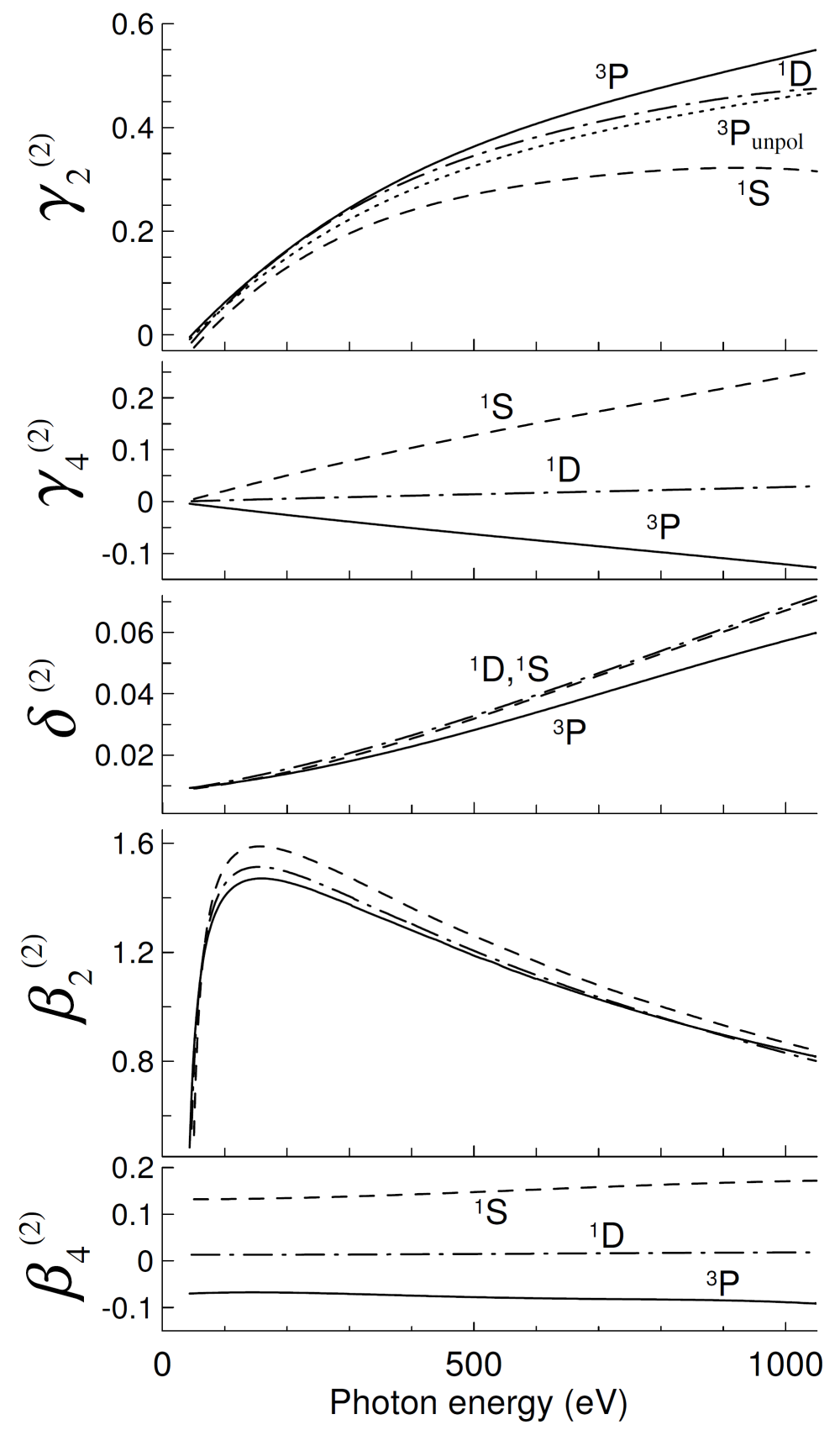

Figure 2. Parameters of the angular distribution of photoelectrons $e_{2}(19)$ emitted in sequential 2PDI of the neon 2p-shell for different terms of the residual ion $\mathrm{Ne}^{++}\left(2 p^{4}\right)$ as a function of the photon energy. Solid curves: ${ }^{3} P$-term; dashed curves: ${ }^{1} S$-term; dashed-dotted curves: ${ }^{1} D$-term. The dotted curve on the upper panel corresponds to the ${ }^{3} P$-term when the intermediate ionic $\mathrm{Ne}^{+}\left(2 p^{52} P\right)$ state is unpolarized. 
average radial electron wave functions. Then the non-dipole parameters in equation (19) are expressed in terms of the single-electron dipole $\left(d_{s}, d_{d}\right)$ and quadrupole $\left(q_{p}, q_{f}\right)$ amplitudes for transitions of the $2 p$ electron into $s, d$ and $p, f$ electron states in the continuum, respectively:

$$
\begin{aligned}
& \delta^{(2)}\left(L_{f} S_{f}\right)=\left(N_{2}^{L_{f} S_{f}}\right)^{-1}\left(1-K_{L_{f} S_{f}} \mathcal{A}_{20}\right) \\
& \times \Im\left[\left(\sqrt{2} d_{s}-d_{d}\right)\left(\sqrt{3} q_{p}^{*}-\sqrt{2} q_{f}^{*}\right)\right], \\
& \gamma_{2}^{(2)}\left(L_{f} S_{f}\right)=\left(N_{2}^{L_{f} S_{f}}\right)^{-1}\left[\Im\left(6 \sqrt{3} d_{d} q_{p}^{*}+2\left(5 d_{s}+2 \sqrt{2} d_{d}\right) q_{f}^{*}\right)\right. \\
&\left.-K_{L_{f} S_{f}} \frac{\mathcal{A}_{20}}{2} \Im\left(3 \sqrt{3} d_{d} q_{p}^{*}+4\left(5 d_{s}-7 \sqrt{2} d_{d}\right) q_{f}^{*}\right)\right], \\
& \gamma_{4}^{(2)}\left(L_{f} S_{f}\right)=-\frac{45 \sqrt{2}}{2}\left(N_{2}^{L_{f} S_{f}}\right)^{-1} K_{L_{f} S_{f}} \mathcal{A}_{20} \Im\left(d_{d} q_{f}^{*}\right), \\
& \beta_{2}^{(2)}\left(L_{f} S_{f}\right)=\left(N_{2}^{L_{f} S_{f}}\right)^{-1}\left[\sqrt{2}\left(d_{s} d_{d}^{*}+d_{d} d_{s}^{*}\right)+\left|d_{d}\right|^{2}-\right. \\
&\left.-K_{L_{f} S_{f}} \mathcal{A}_{20}\left(\sqrt{2}\left(d_{s} d_{d}^{*}+d_{d} d_{s}^{*}\right)+\frac{5}{14}\left|d_{d}\right|^{2}\right)\right], \\
& \beta_{4}^{(2)}\left(L_{f} S_{f}\right)=-\frac{54}{35}\left(N_{2}^{L_{f} S_{f}}\right)^{-1} K_{L_{f} S_{f}} \mathcal{A}_{20}\left|d_{d}\right|^{2},
\end{aligned}
$$

where

$$
N_{2}^{L_{f} S_{f}}=\left|d_{s}\right|^{2}+\left|d_{d}\right|^{2}-K_{L_{f} S_{f}} \mathcal{A}_{20} \frac{1}{10}\left(\left|d_{d}\right|^{2}+10\left|d_{s}\right|^{2}\right)
$$

$K_{1_{S}}=1, K_{1_{D}}=1 / 10, K_{3 P}=-1 / 2$. The single particle dipole and quadrupole amplitudes are of the form

$$
\begin{aligned}
& d_{l}=\sqrt{3}(10,10 \mid l 0) e^{i \delta_{l}} \int P_{2 p}(r) P_{E l}(r) r d r, \\
& q_{l}=\sqrt{3}(10,20 \mid l 0) \sqrt{\frac{3}{5}} \frac{i \alpha \omega}{2 \sqrt{3}} e^{i \delta_{l}} \int P_{2 p}(r) P_{E l}(r) r^{2} d r,
\end{aligned}
$$

respectively, where $P_{2 p}(r)$ and $P_{E l}(r)$ are the electron wave functions in the $2 p$ state and in the continuum of $\mathrm{Ne}^{+}, \delta_{l}$ is the corresponding scattering phase. To obtain (24)-(29) the reduction formula was used

$$
T_{L_{i}, L_{f} l_{2} L_{t_{2}}}^{\pi_{2} L_{2}}=\hat{L}_{i} \hat{L}_{t_{2}}(-1)^{L_{f}+l_{2}+L_{i}}\left\{\begin{array}{ccc}
L_{2} & L_{i} & L_{t_{2}} \\
L_{f} & l_{2} & 1
\end{array}\right\} R_{L_{2}, l_{2}},
$$

where $R_{1, l_{2}}=d_{l_{2}}$ and $R_{2, l_{2}}=q_{l_{2}}$. Equations (24)-(29) are given for the sequential 2PDI via the $\mathrm{Ne}^{+}\left(2 p^{5} P_{3 / 2}\right)$ state and $\mathcal{A}_{20}$ is the integral alignment of this state,

$$
\mathcal{A}_{20}=\rho_{20}\left(J_{i}, J_{i}\right) / \rho_{00}\left(J_{i}, J_{i}\right),
$$

produced in the first ionization step (22). Our results for the integral alignment of the $\mathrm{Ne}^{+}\left(2 p^{2}{ }^{2} P_{3 / 2}\right)$ state are in agreement with the preceding calculations $[13,45]$; it is not 
large and its value smoothly changes in the interval between -0.15 and $-0.20 \ddagger$ over the considered broad energy range.

For ionization via the $\mathrm{Ne}^{+}\left(2 p^{52} P_{1 / 2}\right)$ state, $N_{2}^{L_{f} S_{f}}$ is two times smaller and the alignment $\mathcal{A}_{20}$ vanishes. After incoherent summation over the fine structure $\mathrm{Ne}^{+}\left(2 p^{5}{ }^{2} P_{1 / 2,3 / 2}\right)$ levels, equations (24), (25), (27), (29) remain valid with the reduced values of the coefficients: $K_{1_{S, a v}}=2 / 3, K_{1_{D, a v}}=2 / 30, K_{{ }, a v}=-1 / 3$.

It is obvious from equations (24)-(29) that for an unpolarized intermediate state $\left(\mathcal{A}_{20}=0\right), \delta^{(2)}$ and $\gamma_{2}^{(2)}$ coincide for all $L_{f} S_{f}$ terms of the residual ion $\mathrm{Ne}^{++}\left(2 p^{4}\right)$ and $\beta_{4}^{(2)}=\gamma_{4}^{(2)}=0$. Therefore the main difference between the photoelectron $\left(e_{2}\right)$ angular distributions corresponding to different final ionic terms $\mathrm{Ne}^{++}\left(2 p^{4}{ }^{2 S_{f}+1} L_{f}\right)$ is caused by the alignment of the intermediate ion $\mathrm{Ne}^{+}\left(2 p^{5}\right)$, produced in the first ionization step. Further deviations arise due to the term dependence of the radial electron wavefunctions, but with much smaller effects than the alignment of the intermediate ion. Thus in figure 2 we present for comparison only one curve (in the upper panel) corresponding to the unpolarized intermediate ion. The parameters $\gamma_{2}^{(2)}$ and $\delta^{(2)}$ are close to the corresponding single-photon parameters $\gamma$ and $\delta$ (figure 1). This can be understood by taking into account that the dynamics of the 2 p-ionization from $\mathrm{Ne}\left(2 p^{6}\right)$ and $\mathrm{Ne}^{+}\left(2 p^{5}\right)$ is similar and the aligment of the $\mathrm{Ne}^{+}\left(2 p^{5}\right)$ state is small. Generally, effects caused by the alignment of the intermediate ion $\mathrm{Ne}^{+}\left(2 p^{5}\right)$ (i.e. the parameters $\gamma_{4}^{(2)}\left(L_{f} S_{f}\right)$ and $\left.\beta_{4}^{(2)}\left(L_{f} S_{f}\right)\right)$ are strongest for ionization to the ${ }^{1} S$ term of the residual ion $\mathrm{Ne}^{++}\left(2 p^{4}\right)$. For example the absolute value of $\gamma_{4}^{(2)}$ for the ${ }^{1} S$ residual ion state is ten and two times larger than for the ${ }^{1} D$ and ${ }^{3} P$ states, respectively. Note that it is possible to write down the expression for $\delta^{(2)}$ in the product form (24), where dipole and quadrupole amplitudes are separated in different factors. The dipole factor $\left(\sqrt{2} d_{s}-d_{d}\right)$ is accidentally close to zero due to mutual cancellation of the two dipole amplitudes in the broad energy range. This is a reason for the small values of $\delta^{(2)}$ even at energies where the non-dipole ionization transitions are prominent.

The non-dipole effects violate the axial symmetry with respect to the linear polarization vector of the photon beam, or, equivalently, result in a forward-backward asymmetry with respect to the photon propagation vector. The forward-backward asymmetry is defined as

$$
A(\theta)=\frac{W(\theta)-W(\pi-\theta)}{W(\theta)+W(\pi-\theta)},
$$

where $W(\theta)$ is the photoelectron flux emitted into the angle $\theta$ given relative to the direction of the photon propagation in the plane spanned by the direction of the propagation and the electric field of the linearly polarized photon. The asymmetry is a convenient quantity to study the non-dipole effects [46], since $A(\theta)=0$ in the dipole approximation. Our numerical analysis for neon shows that for $\theta=45^{\circ}$ the values of the asymmetry are almost maximal in comparison with asymmetries for other

$\ddagger$ Kleiman and Lohmann [45] use a coordinate system with the $z$-axis along the radiation beam. The value of the alignment from [45] is related to the present one as $\mathcal{A}_{20}^{\text {present }}=-2 \mathcal{A}_{20}^{\mathrm{KL}}$. 


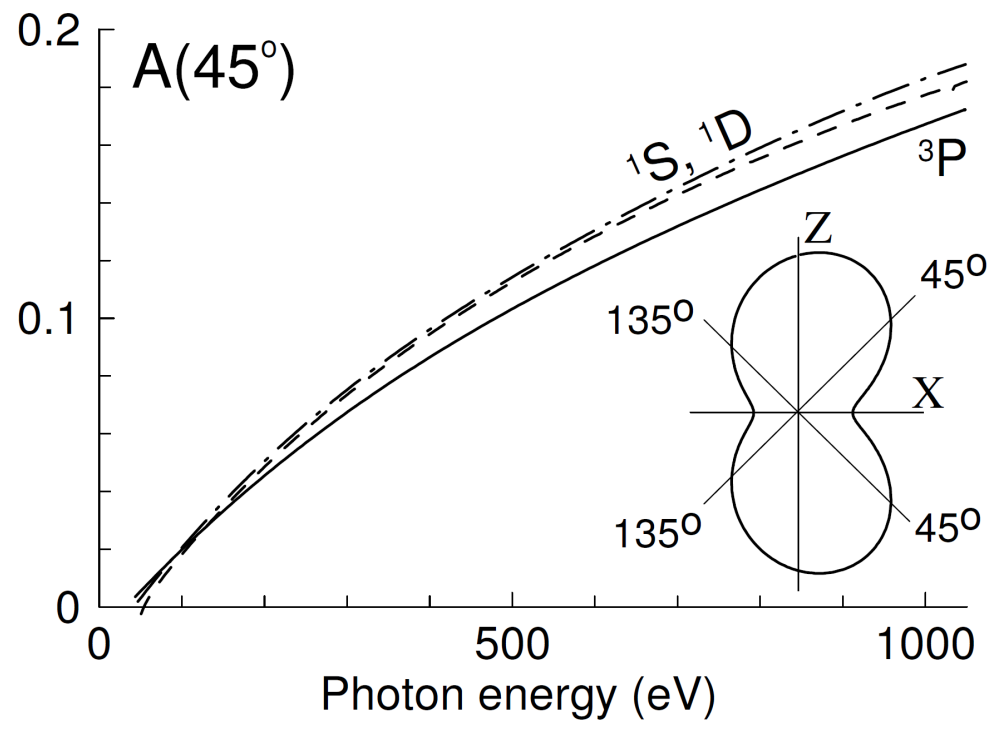

Figure 3. Asymmetry (34) for $\theta=45^{\circ}$ as a function of the photon energy for different final multiplet states of $\mathrm{Ne}^{++}\left(2 p^{4}\right)$. Curves as in figure 2. The inset shows the angular distribution of the $e_{2}$ photoelectrons at $1 \mathrm{keV}$ photon energy for the ${ }^{3} P$-term of the ion $\mathrm{Ne}^{++}\left(2 p^{4}\right)$. Axis $x$ is along the linearly polarized photon beam, axis $z$ is along the electric field of the photon.

values of $\theta$. Figure 3 demonstrates that $A\left(45^{\circ}\right)$ only weakly depends on the term of the final ionic state and monotonously increases with the photon energy approaching $20 \%$ at the energies around $1 \mathrm{keV}$. For these values of the asymmetry, the electron flux ratio $W\left(45^{\circ}\right) / W\left(135^{\circ}\right)$ reaches the value of 1.4 . For the experimental observation of the non-dipole contribution it is not necessary to resolve the final multiplets of $\mathrm{Ne}^{++}\left(2 p^{4}\right)$ to observe the asymmetry (34).

\section{Conclusions}

We obtained general expressions for the angular distributions of photoelectrons in the sequential two-photon atomic double ionization by accounting for the full multipole expansion of the radiation field. The development provides grounds for treating this non-linear process at photon energies accessible by X-ray free electron lasers, i.e. where the dipole approximation regularly breaks down. The general formalism is applied to derive more particular expressions for the photoelectron angular distributions within the first-order non-dipole corrections. The role of the alignment of the intermediate ionic state, produced in the first ionization step, is crucial for the appearing of new dynamical parameters in comparison with one-photon single ionization. Our numerical calculations for the photon energies up to $1 \mathrm{keV}$ demonstrate that the non-dipole effects in the sequential two-photon 2p-ionization of neon can be observed with existing FEL facilities. The influence of longitudinal coherence of the FEL pulses on the non-dipole effects is an important issue for further studies. 
Non-dipole effects in sequential two-photon double ionization

\section{Acknowledgments}

ANG and EVG acknowledge support by the Russian Foundation for Basic Research (RFBR) under grant 12-02-01123 and the hospitality of the European XFEL GmbH, where this work was completed. EVG gratefully acknowledges financial support by the Russian President grant MK-6509.2012.2 and by the Dynasty Foundation via the Support Program for Young Scientists. ANG acknowledges a support by the program "Physics with Accelerators and Reactors in West Europe" of the Russian Ministry of Education and Science. This work is part of the project Correlation and Polarization Phenomena in Ionization of Dilute Species by XUV and X-ray Radiation in the framework of the German-Russian collaboration "Development and Use of AcceleratorBased Photon Sources".

\section{Appendix}

In this Appendix we use the following notations for brevity:

$$
\begin{gathered}
B_{k_{1} k_{i} k_{\gamma_{1}}} \equiv \Re B^{E 1, E 1}\left(k_{1}, k_{i}, k_{\gamma_{1}}\right), \\
B_{k_{1} k_{i} k_{\gamma_{1}}}^{E} \equiv \Re B^{E 1, E 2}\left(k_{1}, k_{i}, k_{\gamma_{1}}\right), \quad B_{k_{1} k_{i} k_{\gamma_{1}}}^{M} \equiv \Re B^{E 1, M 1}\left(k_{1}, k_{i}, k_{\gamma_{1}}\right), \\
B_{k_{1} k_{i} k_{\gamma_{1}}}^{E-M} \equiv B_{k_{1} k_{i} k_{\gamma_{1}}}^{E}-B_{k_{1} k_{i} k_{\gamma_{1}}}^{M}, \quad B_{k_{1} k_{i} k_{\gamma_{1}}}^{E+M} \equiv B_{k_{1} k_{i} k_{\gamma_{1}}}^{E}+\frac{3}{\sqrt{5}} B_{k_{1} k_{i} k_{\gamma_{1}}}^{M}
\end{gathered}
$$

and similar for the coefficients $\bar{B}_{k_{i} k_{2} k_{\gamma_{2}}}$.

When the sequential $2 \mathrm{PDI}$ proceeds via the ${ }^{2} P_{3 / 2}$ state, the angular distribution of the electron $e_{1}$ is determined by the parameters

$$
\begin{aligned}
\beta_{2}^{(1)} & =-N_{1}^{-1} \frac{\sqrt{2}}{35}\left[35 \bar{B}_{000} B_{202}+\bar{B}_{202}\left(7 B_{220}+2 \sqrt{35} B_{222}\right)\right], \\
\beta_{4}^{(1)} & =N_{1}^{-1} \frac{2 \sqrt{2}}{\sqrt{35}} \bar{B}_{202} B_{422}, \\
\delta^{(1)} & =N_{1}^{-1}\left\{-\bar{B}_{000}\left[\sqrt{6} B_{101}^{E-M}+2 B_{303}^{E}\right]+\sqrt{3} \bar{B}_{202}\left[\frac{\sqrt{2}}{5} B_{121}^{E-M}-\frac{\sqrt{2}}{3} B_{122}^{E+M}\right.\right. \\
& \left.\left.-\frac{8 \sqrt{2}}{15} B_{123}^{E}-\frac{3 \sqrt{7}}{35} B_{321}^{E-M}-\frac{\sqrt{42}}{42} B_{322}^{E+M}-\frac{\sqrt{2}}{5} B_{323}^{E}-\frac{\sqrt{11}}{11} B_{523}^{E}\right]\right\}, \\
\gamma_{2}^{(1)} & =N_{1}^{-1}\left[10 \bar{B}_{000} B_{303}^{E}\right. \\
& \left.+\bar{B}_{202}\left(\frac{3 \sqrt{21}}{7} B_{321}^{E-M}+\frac{5 \sqrt{14}}{14} B_{322}^{E+M}+\sqrt{6} B_{323}^{E}+\frac{14 \sqrt{33}}{11} B_{523}^{E}\right)\right], \\
\gamma_{4}^{(1)} & =-N_{1}^{-1} \frac{21 \sqrt{3}}{\sqrt{11}} \bar{B}_{202} B_{523}^{E},
\end{aligned}
$$


where $N_{1}=\bar{B}_{000}+\frac{2}{\sqrt{5}} \bar{B}_{202} B_{022}$. The angular distribution of the electron $e_{2}$ is determined by the parameters

$$
\begin{aligned}
\beta_{2}^{(2)}= & N_{2}^{-1}\left[\mathcal{A}_{20}\left(\bar{B}_{220}+\frac{2}{\sqrt{7}} \bar{B}_{222}\right)-\sqrt{2} \bar{B}_{022}\right] \\
\beta_{4}^{(2)}= & -N_{2}^{-1} \frac{6}{\sqrt{35}} \mathcal{A}_{20} \bar{B}_{242}, \\
\delta^{(2)}= & N_{2}^{-1}\left[-\sqrt{6} \bar{B}_{011}^{E-M}-2 \bar{B}_{033}^{E}+\mathcal{A}_{20}\left(-\bar{B}_{212}^{E+M}-\sqrt{\frac{3}{5}} \bar{B}_{211}^{E-M}\right.\right. \\
& \left.\left.+\frac{3}{\sqrt{10}} \bar{B}_{231}^{E-M}-\frac{1}{2} \bar{B}_{232}^{E+M}+\frac{8}{\sqrt{35}} \bar{B}_{213}^{E}+\sqrt{\frac{3}{5}} \bar{B}_{233}^{E}+\sqrt{\frac{15}{14}} \bar{B}_{253}^{E}\right)\right] \\
\gamma_{2}^{(2)}= & N_{2}^{-1}\left[10 \bar{B}_{033}^{E}+\mathcal{A}_{20} \sqrt{5}\left(\frac{\sqrt{5}}{2} \bar{B}_{232}^{E+M}-\frac{3 \sqrt{2}}{2} \bar{B}_{231}^{E-M}\right.\right. \\
& \left.\left.-\sqrt{3} \bar{B}_{233}^{E}-\sqrt{42} \bar{B}_{253}^{E}\right)\right], \\
\gamma_{4}^{(2)}= & N_{2}^{-1} \frac{3 \sqrt{105}}{\sqrt{2}} \mathcal{A}_{20} \bar{B}_{235}^{E},
\end{aligned}
$$

where $N_{2}=\bar{B}_{000}-\sqrt{\frac{2}{5}} \mathcal{A}_{20} \bar{B}_{202}$ and the integral alignment $\mathcal{A}_{20}$ is defined by equation (33).

For the $2 \mathrm{PDI}$ via the ${ }^{2} P_{1 / 2}$ state, $\beta_{4}^{(1)}=\gamma_{4}^{(1)}=\beta_{4}^{(2)}=\gamma_{4}^{(2)}=0$ and all terms with $\bar{B}_{202}$ vanish in the expressions for the parameters $\beta_{2}^{(1)}, \delta^{(1)}, \gamma_{2}^{(1)}$ as well as for $N_{1}$, while in the expressions for the parameters $\beta_{2}^{(2)}, \delta^{(2)}, \gamma_{2}^{(2)}$ as well as for $N_{2}$, all terms with $\mathcal{A}_{20}$ vanish.

\section{References}

[1] Berrah N et al 2010 J. Mod. Opt. 571015

[2] Bachau H and Lambropoulos P 1991 Phys. Rev. A 44 R9

[3] Laulan S and Bachau H 2003 Phys. Rev. A 68013409

[4] Delone N B and Krainov V P 1985 Atoms in Strong Light Fields (Berlin: Springer-Verlag)

[5] Faisal F H M 1986 Theory of Multiphoton Processes (New York and London: Plenum Press)

[6] Mittleman M H 1993 Introduction to the Theory of Laser-Atom interactions (2nd ed) (New York and London: Plenum Press)

[7] Rand S C 2010 Nonlinear and Quantum Optics Using the Density Matrix (Oxford: Oxford University Press)

[8] Lambropoulos P, Doolen G, Rountree S P 1975 Phys. Rev. Lett. 34636

[9] Dodhy A, Compton R N and Stockdale J A D 1986 Phys. Rev. A 332167

[10] Lyras A et al 1988 Phys. Rev. A 37403

[11] Lépine F, Zamith S, de Snaijer A, Bordas Ch and Vrakking M J J 2004 Phys. Rev. Lett. 93233003

[12] Kheifets A S 2007 J. Phys. B: At. Mol. Opt. Phys. 40 F313

[13] Fritzsche S, Grum-Grzhimailo A N, Gryzlova E V and Kabachnik N M 2008 J. Phys. B: At. Mol. Opt. Phys. 41165601 
[14] Gryzlova E V, Grum-Grzhimailo A N, Fritzsche S and Kabachnik N M 2010 J. Phys. B: At. Mol. Opt. Phys. 43225602

[15] Braune M, Reinköster A, Viefhaus J, Lohmann B and Becker U 2007 XXV Int. Conf. on Photonic, Electronic and Atomic Collisions (ICPEAC) (Freiburg, Germany) Book of abstracts, Fr034

[16] Kurka et al 2009 J. Phys. B 42141002

[17] Fukuzawa H et al 2010 J. Phys. B. 43111001

[18] Hikosaka Y et al 2010 Phys. Rev. Lett. 105133001

[19] Miyauchi N et al 2011 J. Phys. B 44071001

[20] Rouzée A et al 2011 Phys. Rev. A 83 031401(R)

[21] Gryzlova E V et al 2011 Phys. Rev. A, 84063405

[22] Braune M, Lischke T, Meißner A, Ilchen M, Deinert S, Viefhaus J, Knie A and Becker U 2011 XXVII Int. Conf. on Photonic, Electronic and Atomic Collisions (ICPEAC) (Belfast, Northern Ireland, UK) Abstracts, TU110 and private communication

[23] Hemmers O, Guillemin R and Lindle D W 2004 Radiat. Phys. Chem. 70123

[24] Guillemin R, Hemmers O, Lindle D W and Manson S T 2006 Radiat. Phys. Chem. 752258

[25] Trzhaskovskaya M B, Nefedov V I and Yarzhemsky V G 2001 Atomic Data and Nuclear Data Tables $\mathbf{7 7} 97$

[26] Balashov V V, Grum-Grzhimailo A N and Kabachnik N M 2000 Polarization and Correlation Phenomena in Atomic Collisions. A Practical Theory Course (New York: Kluwer Plenum)

[27] Grum-Grzhimailo A N 2003 J. Phys. B: At. Mol. Opt. Phys. 362385

[28] Grum-Grzhimailo A N 2001 J. Phys. B: At. Mol. Opt. Phys. 34 L359

[29] Hansen J C, Duncanson J A Jr, Chien R-L and Berry R S 1980 Phys. Rev. A 21222

[30] Chien R-L, Mullins O C and Berry R S 1983 Phys. Rev. A 282078

[31] Fritzsche S, Grum-Grzhimailo A N, Gryzlova E V and Kabachnik N M 2011 J. Phys. B: At. Mol. Opt. Phys. 44175602

[32] Grum-Grzhimailo A N, Gryzlova E V, Strakhova S I, Kabachnik N M and Fritzsche S $2009 \mathrm{~J}$. Phys: Conf. Ser. 194012004

[33] Fritzsche S, Grum-Grzhimailo A N, Gryzlova E V and Kabachnik N M 2009 J. Phys. B: At. Mol. Opt. Phys. 42145602

[34] Cooper J W 1993 Phys. Rev. A 471841

[35] Becker U and Shirley D A 1996 In: VUV and Soft X-ray Photoionization eds U Becker and D A Shirley (New York and London: Plenum Press) p. 135

[36] Hemmers O et al 1997 J. Phys. B: At. Mol. Opt. Phys. 30 L727

[37] Derevianko A, Hemmers O, Oblad S, Glans P, Wang H, Whitfield S B, Wehlitz R, Sellin I A, Johnson W R and Lindle D W 2000 Phys. Rev. Lett. 842116

[38] Rudenko A et al 2008 Phys. Rev. Lett. 101073003

[39] Kheifets A S 2009 J. Phys. B: At. Mol. Opt. Phys. 42134016

[40] Johnson W R, Derevianko A, Cheng K T, Dolmatov V K and Manson S T 1999 Phys. Rev. A 59 3609

[41] Derevianko A, Johnson W R and Cheng K T 1999 Atomic Data and Nuclear Data Tables 73153

[42] Kanter E P et al 2011 Phys. Rev. Lett. 107233001

[43] Geloni G, Kocharyan V and Saldin E 2011 J. Mod. Opt. 581391 and 2012 DESY Report 12-034

[44] Schlotter W F et al 2012 Rev. Sci. Instrum. 83043107

[45] Kleiman U and Lohmann B 2003 J. Electron Spectrosc. Relat. Phenom. 131-132 29

[46] Krässig B, Bilheux J-C, Dunford R W, Gemmell D S, Hasegawa S, Kanter E P, Southworth S H, Young L, LaJohn L A and Pratt R H 2003 Phys. Rev. A 67022707 